\title{
Dense quark matter in compact stars
}

\author{
M. Alford \\ Physics Department \\ Washington University CB 1105 \\ Saint Louis, MO 63130 \\ USA
}

29 May 2003

\begin{abstract}
The densest predicted state of matter is colour-superconducting quark matter, in which quarks near the Fermi surface form a condensate of Cooper pairs. This form of matter may well exist in the core of compact stars, and the search for signatures of its presence is an ongoing enterprise. Using a bag model of quark matter, I discuss the effects of colour superconductivity on the mass-radius relationship of compact stars, showing that colour superconducting quark matter can occur in compact stars at values of the bag constant where ordinary quark matter would not be allowed. The resultant "hybrid" stars with colour superconducting quark matter interior and nuclear matter surface have masses in the range 1.3-1.6 $M_{\odot}$ and radii 8-11 km. Once perturbative corrections are included, quark matter can show a mass-radius relationship very similar to that of nuclear matter.
\end{abstract}




\section{Introduction}

It has become clear over the last few years that the phase diagram of QCD is much richer than originally believed. In addition to the hadronic phase with which we are familiar and the quark gluon plasma (QGP) that is predicted to lie at temperatures above $170 \mathrm{MeV}$, there is a whole family of "colour superconducting" phases, that are expected to occur at high density and low temperature [1. The essence of colour superconductivity is quark pairing, driven by the BCS mechanism, which operates when there exists an attractive interaction between fermions at a Fermi surface. The QCD quark-quark interaction is strong, and is attractive in many channels, so we expect cold dense quark matter to generically exhibit colour superconductivity. Moreover, quarks, unlike electrons, have colour and flavour as well as spin degrees of freedom, so many different patterns of pairing are possible. This leads us to expect a rich phase structure in matter beyond nuclear density.

Colour superconducting quark matter may occur naturally in the universe, in the cold dense cores of neutron stars. (It may also be possible to create it in low-energy heavy ion colliders, such as the proposed Compressed Baryonic Matter facility at GSI Darmstadt.) There densities are above nuclear density, and temperatures are of the order of tens of keV. Thus most work on signatures has focussed on properties of colour superconducting quark matter that would affect observable features of compact stars, and I will discuss some of these below.

\section{Phase diagram of QCD}

The strange quark plays a crucial role in the phases of QCD. In Fig. 11 I show two conjectured phase diagrams for QCD, one for a light strange quark and one for a heavy strange quark. In both cases, along the horizontal axis the temperature is zero, and the density rises from the onset of nuclear matter through the transition to quark matter. Compact stars are in this region of the phase diagram, although it is not known whether their cores are dense enough to reach the quark matter phase. Along the vertical axis the temperature rises, taking us through the crossover from a hadronic gas to the quark gluon plasma. This is the regime explored by high-energy heavy-ion colliders (see other contributions to these proceedings).

If the effective strange quark mass is low enough in quark matter at a few times nuclear density, then there is a direct transition from nuclear matter to colourflavour-locked (CFL) quark matter [2]. In the CFL phase the strange quark participates symmetrically with the up and down quarks in Cooper pairing - this is described in more detail below. If the strange quark is too heavy to pair symmetrically with the light quarks at these densities, then there will be an interval of some other phase. This may be crystalline colour superconductivity [3] or some form of single-flavour pairing [4]. 
Heavy strange quark

Light strange quark
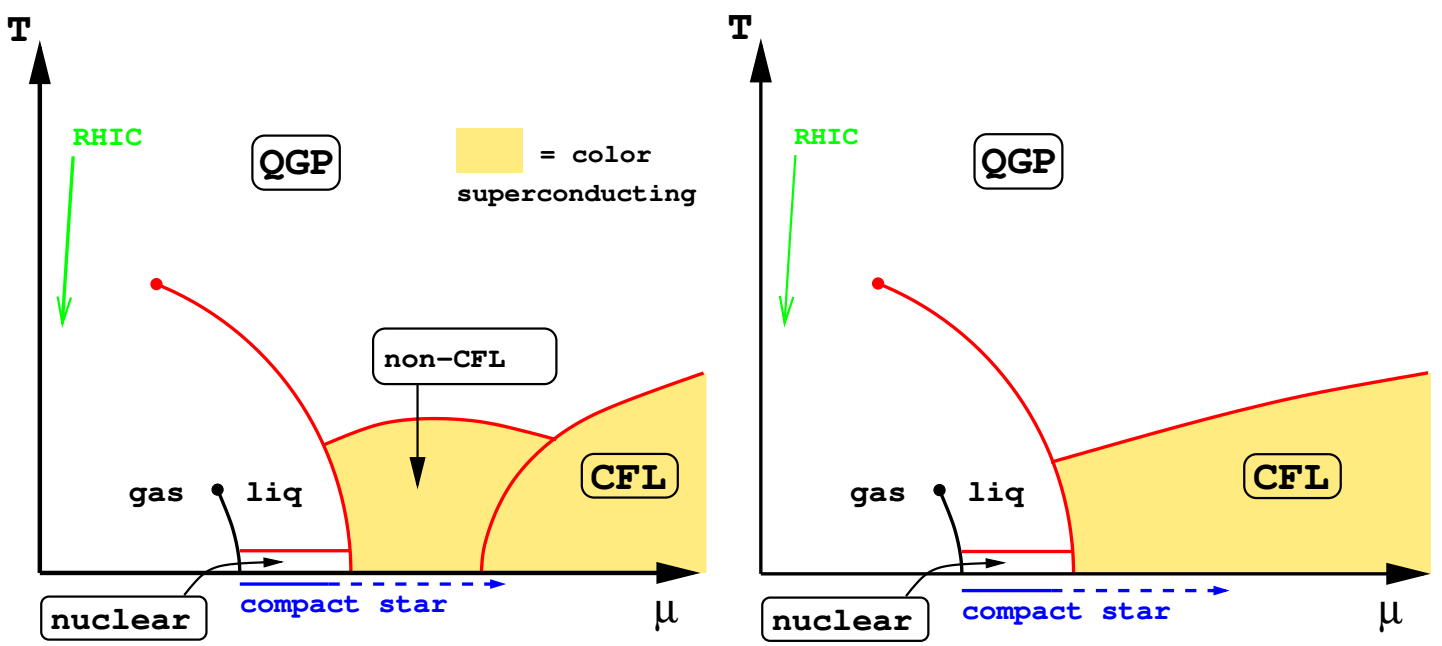

Figure 1: Conjectured phase diagrams for QCD in the real world. For a light strange quark, there is a direct transition from nuclar matter to colour-flavour locked colour superconducting quark matter. For a heavy strange quark, there is an intermediate phase where the strange quark pairs in some other way. Depending on the strength of instanton interactions, the CFL phase may include $K^{0}$ condensation.

\section{Colour superconductivity}

To understand the essence of colour superconductivity, we can borrow some intuition from condensed matter physics [5, 6]. QCD is asymptotically free - the interaction becomes weaker as the momentum grows - so at sufficiently high density and low temperature, there is a Fermi surface of almost free quarks. The interactions between quarks near the Fermi surface are certainly attractive in some channels (quarks bind together to form baryons) and it was shown by Bardeen, Cooper, and Schrieffer (BCS) [7] that if there is any channel in which the interaction is attractive, then there is a state of lower free energy than a simple Fermi surface. That state arises from a complicated coherent superposition of pairs of particles (and holes) - "Cooper pairs".

We can understand the BCS mechanism in an intuitive way as follows. The Helmholtz free energy is $F=E-\mu N$, where $E$ is the total energy of the system, $\mu$ is the chemical potential, and $N$ is the number of fermions. The Fermi surface is defined by a Fermi energy $E_{F}=\mu$, at which the free energy is minimized, so adding or subtracting a single particle costs zero free energy. Now switch on a weak attractive interaction. It costs no free energy to add a pair of particles (or holes), and the attractive interaction between them then lowers the free energy of the system. Many such pairs will therefore be created in the modes near the Fermi surface, and these pairs, being bosonic, will form a condensate. The ground state 
will be a superposition of states with all numbers of pairs, breaking the fermion number symmetry.

Since pairs of quarks cannot be colour singlets, the resulting condensate will break the local colour symmetry $S U(3)_{\text {colour }}$. We call this "colour superconductivity". Note that the quark pairs play the same role here as the Higgs particle does in the standard model: the colour-superconducting phase can be thought of as the Higgs phase of QCD. For more detailed discussions of calculations of the favored pairing patterns, see the reviews [1].

\subsection{Three flavours: Colour-flavour locking (CFL)}

The favored pairing pattern at high densities, where the strange quark Fermi momentum is close to the up and down quark Fermi momenta, is "colour-flavour locking" (CFL). In QCD with three flavours of massless quarks the Cooper pairs cannot be flavour singlets, and both colour and flavour symmetries are necessarily broken [2. Both NJL [2, 8, 9] and gluon-mediated interaction calculations [10] agree that the attractive channel exhibits a pattern called colour-flavour locking (CFL),

$$
\begin{gathered}
\left\langle q_{i}^{\alpha} q_{j}^{\beta}\right\rangle_{1 P I} \propto C \gamma_{5}\left((\kappa+1) \delta_{i}^{\alpha} \delta_{j}^{\beta}+(\kappa-1) \delta_{j}^{\alpha} \delta_{i}^{\beta}\right) \\
{\left[S U(3)_{\text {colour }}\right] \times \underbrace{S U(3)_{L} \times S U(3)_{R}}_{\supset\left[U(1)_{Q}\right]} \times U(1)_{B} \rightarrow \underbrace{S U(3)_{C+L+R}}_{\supset\left[U(1)_{\tilde{Q}}\right]} \times \mathbb{Z}_{2}}
\end{gathered}
$$

Colour indices $\alpha, \beta$ and flavour indices $i, j$ run from 1 to 3, Dirac indices are suppressed, and $C$ is the Dirac charge-conjugation matrix. The term multiplied by $\kappa$ corresponds to pairing in the $\left(\mathbf{6}_{S}, \mathbf{6}_{S}\right)$, which although not energetically favored breaks no additional symmetries and so $\kappa$ is in general small but not zero [2, 11, 10, The Kronecker deltas connect colour indices with flavour indices, so that the condensate is not invariant under colour rotations, nor under flavour rotations, but only under simultaneous, equal and opposite, colour and flavour rotations. Since colour is only a vector symmetry, this condensate is only invariant under vector flavour+colour rotations, and breaks chiral symmetry. The effect of including a strange quark mass is to distort the CFL pairing [12, 8], and a sufficiently large strange quark mass will unpair the strange quark completely [12, 8, 13, yielding a two-flavour colour superconductor (2SC) 6], and at lower values it may induce a flavour rotation of the condensate known as "kaon condensation" [14]. The features of the CFL pattern of condensation are

- The colour gauge group is completely broken. All eight gluons become massive. This ensures that there are no infrared divergences associated with gluon propagators.

- All the quark modes are gapped. The nine quasiquarks (three colours times three flavours) fall into an $\mathbf{8} \oplus \mathbf{1}$ of the unbroken global $S U(3)$, so there are two gap parameters. The singlet has a larger gap than the octet. 
- A rotated electromagnetism (" $\tilde{Q} ")$ survives unbroken. It is a combination of the original photon and one of the gluons.

- Two global symmetries are broken, the chiral symmetry and baryon number, so there are two gauge-invariant order parameters that distinguish the CFL phase from the QGP, and corresponding Goldstone bosons which are longwavelength disturbances of the order parameter. When the light quark mass is non-zero it explicitly breaks the chiral symmetry and gives a mass to the chiral Goldstone octet, but the CFL phase is still a superfluid, distinguished by its baryon number breaking.

- The symmetries of the 3-flavour CFL phase are the same as those one might expect for 3-flavour hypernuclear matter [8, so it is possible that there is no phase transition between them.

In a real neutron star we must require electromagnetic and colour neutrality [15, 13] (ignoring charge-separated phases). It turns out that this penalizes the $2 \mathrm{SC}$ phase relative to the CFL phase [13. The reason is that the CFL phase has already paid most of the cost of neutrality, since it brings the $u, d$, and $s$ Fermi surfaces close together [13, 16, 17], and the 2SC phase only pairs 4 of the 9 quark colours and flavours, so it has much less pair binding energy than the CFL phase.

The arguments made in Ref. [13] are model-independent, based on simplified assumptions about the dependence of the constituent strange quark mass $M_{s}$ on $\mu$ and expanding the free energy in powers of $M_{s} / \mu$. The NJL calculation of Ref. [17] handles $M_{s} \sim \mu$ and includes the coupling between the chiral condensate and quark condensate gap equations. The net result is that once neutrality is imposed, there is no (or very little) density range in which $2 \mathrm{SC}$ is the phase with the lowest free energy.

\section{Mass-radius relationship for compact stars}

The high density and relatively low temperature required to produce colour superconducting quark matter may be attained in compact stars, also known as "neutron stars", since it is often assumed that they are made primarily of neutrons. Typical compact stars have masses close to $1.4 M_{\odot}$, and are believed to have radii of order $10 \mathrm{~km}$. (For reviews see Ref. [18, 19]).

Colour superconductivity affects the equation of state at order $(\Delta / \mu)^{2}$. It also gives mass to excitations around the ground state: it opens up a gap at the quark Fermi surface, and makes the gluons massive. One would therefore expect it to have a profound effect on transport properties, such as mean free paths, conductivities and viscosities.

Although the effects of colour superconductivity on the quark matter equation of state are subdominant, they may have a large effect on the mass-radius relationship. The reason for this is that the pressure of quark matter relative to the hadronic 
vacuum contains a constant (the "bag constant" $B$ ) that represents the cost of dismantling the chirally broken and confining hadronic vacuum,

$$
p \sim \mu^{4}+\Delta^{2} \mu^{2}-B
$$

If the bag constant is large enough so that nuclear matter is favored (or almost favored) over quark matter at $\mu \sim 320 \mathrm{MeV}$, then the bag constant and $\mu^{4}$ terms almost cancel, and the superconducting gap $\Delta$ may have a large effect on the equation of state and hence on the mass-radius relationship of a compact star.

In Ref. [20] Sanjay Reddy and I explored the effect of quark pairing on the $M-R$ relationship at values of the bag constant that are consistent with nuclear phenomenology. Fig. 2 shows the mass-radius curve for a plausible model of dense matter: the Walecka nuclear equation of state, and quark matter with physically reasonable values of the bag constant $B^{1 / 4}=180 \mathrm{MeV}\left(B=137 \mathrm{MeV} / \mathrm{fm}^{3}\right)$ and strange quark mass $m_{s}=200 \mathrm{MeV}$ [19]. Curves for unpaired $(\Delta=0)$ and coloursuperconducting $(\Delta=100 \mathrm{MeV})$ quark matter are shown. At these values the stars are typically "hybrid", containing both quark matter and nuclear matter. The solid lines in Fig. 2 correspond to stars that either have no QM at all, or a sharp transition between NM and QM: the core is made of quark matter, which is the favored phase at high pressure, and at some radius there is a transition to nuclear matter, which is favored at low pressure. The transition pressure is sensitive to $\Delta$, for reasons discussed earlier. The dashed lines are for stars that contain a mixed NM-QM phase. In all cases we see that light, large stars consist entirely of nuclear matter. When the star becomes heavy enough, the central pressure rises to a level where QM, either in a mixed phase or in its pure form, occurs in the core. As can be seen from the figure the transition density is very sensitive to $\Delta$. 

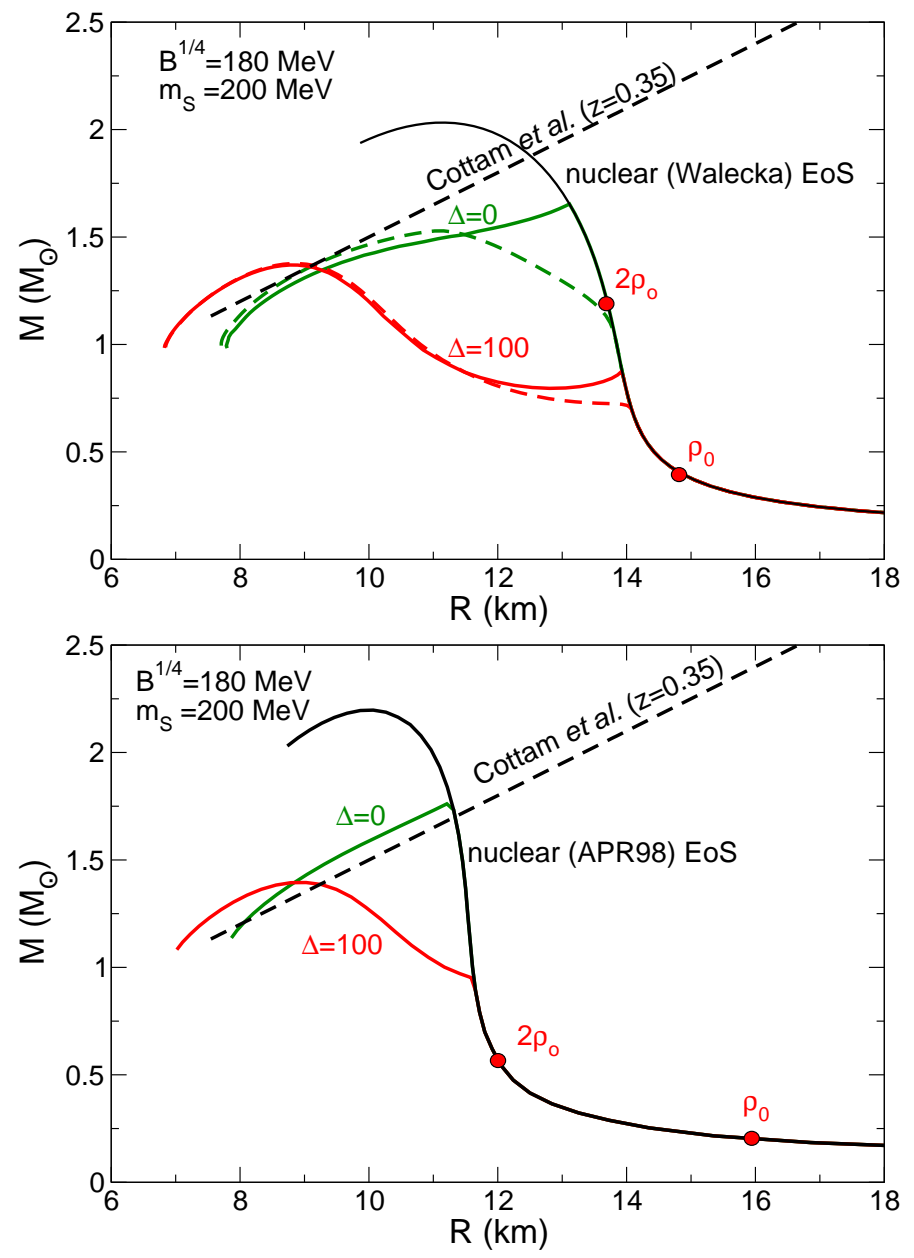

Figure 2: Mass-radius relationships at fixed bag constant $B^{1 / 4}=180 \mathrm{MeV}$ and $m_{s}=200 \mathrm{MeV}$, for unpaired $(\Delta=0)$ and colour-superconducting $(\Delta=100 \mathrm{MeV})$ quark matter. The mixed phase (dashed) and the sharp interface curves are shown. The line labeled "Cottam et al." indicates the constraint obtained by recent measurements of the redshift on three spectral lines from EXO0748-676 [21]. The dots labeled $\rho_{0}$ and $2 \rho_{0}$ on the nuclear matter mass-radius curve indicate that the central density at these locations correspond to nuclear and twice nuclear saturation density respectively. The top panel uses the Walecka equation of state for nuclear matter, and the lower panel uses APR98 (in which case we only consider the sharp-interface scenario). 
In Ref. [20] we kept the bag constant fixed, assuming that it could be fixed by other observations, and we treated the quark matter as free quarks with a pairing energy. It is interesting to see what happens when we relax these assumptions. To see how closely quark matter can mimic nuclear matter, we tune the bag constant to keep constant the density of nuclear matter at its transition to quark matter. To allow for effects of quark interactions beyond Cooper pairing, we follow the parameterization of Fraga et. al. [22], who find that the $\varnothing\left(\alpha_{s}^{2}\right)$ pressure for three flavours over a finite interval in $\mu$ can be mimicked by a bag model inspired form given by

$$
\begin{aligned}
P_{\alpha_{s}^{2}}\left(\mu_{\mathrm{low}} \leq \mu \leq \mu_{\mathrm{high}}\right) & =\frac{3}{4 \pi^{2}} a_{\mathrm{eff}} \mu^{4}-B_{\mathrm{eff}} \\
a_{\mathrm{eff}} & \equiv 1-c
\end{aligned}
$$

Choosing the renormalization scale $\Lambda=2 \mu$ they find that $a_{\text {eff }}=0.628(c=0.372)$ and $B_{\text {eff }}^{1 / 4}=223 \mathrm{MeV}$ in the range $\mu=425$ to $650 \mathrm{MeV}$ and for $\Lambda=3 \mu$ they find that $a_{\mathrm{eff}}=0.626(c=0.374)$ and $B_{\mathrm{eff}}^{1 / 4}=157 \mathrm{MeV}$ in the range $\mu=300$ to 470 $\mathrm{MeV}$.

First, we explore the effect of a colour superconducting gap $\Delta$ and perturbative correction $c$ on the mass-radius relationship. We fix the bag constant by requiring that that the nuclear to quark matter phase transition occur at nuclear matter baryon density $\rho=1.5 n_{\text {sat }}$. The resultant $M(R)$ curves are shown in Fig. 3,

1. The stars resulting from quark matter equations of state without perturbative correction ( $c=0$,blue lines) are smaller and lighter. This is because they are inherently highly favored, and so requiring the phase transition to occur at $\rho=1.5 n_{\text {sat }}$ leads to a large bag constant. By contrast, the equations of state with perturbative correction ( $c=0.4$, red lines) are inherently less favored, and require a low bag constant to bring the phase transition down to $\rho=1.5 n_{\text {sat }}$.

2. The stars with $c=0.3$ have mass-radius relationships that are very similar to the pure nuclear APR98 matter. In fact, for the case where there are perturbative corrections but no colour superconductivity the equations of state $(p(\mu))$ are so similar that our program found a series of phase transitions back and forth between CFL and APR98 up to $\mu=546 \mathrm{MeV}$ (baryon density $\left.\rho=5.4 n_{\text {sat }}\right)$. This is why the $c=0.3$ red dotted curve lies almost exactly on top of the solid black (APR98) curve, even though there was a phase transition from APR98 to CFL at $\rho=1.5 n_{\text {sat }}$ (which is first attained when the APR98 star reaches a mass of $\left.0.315 M_{\odot}, R=13.3 \mathrm{~km}\right)$.

3. In Ref. [20] we showed that at fixed bag constant, colour superconductivity has a strong effect on the mass-radius relationship of compact stars. Here, by comparing the dashed lines with the dotted lines in Fig. 3. we see that it is difficult to distinguish the effect of colour superconductivity from a change in the bag constant. In Fig. 3, as we vary parameters $c$ and $\Delta$ of the quark 
matter equation of state, the bag constant is tuned to maintain a constant value of the nuclear density at the transition to quark matter, and in this situation colour superconductivity only makes a small difference to the massradius relationship.

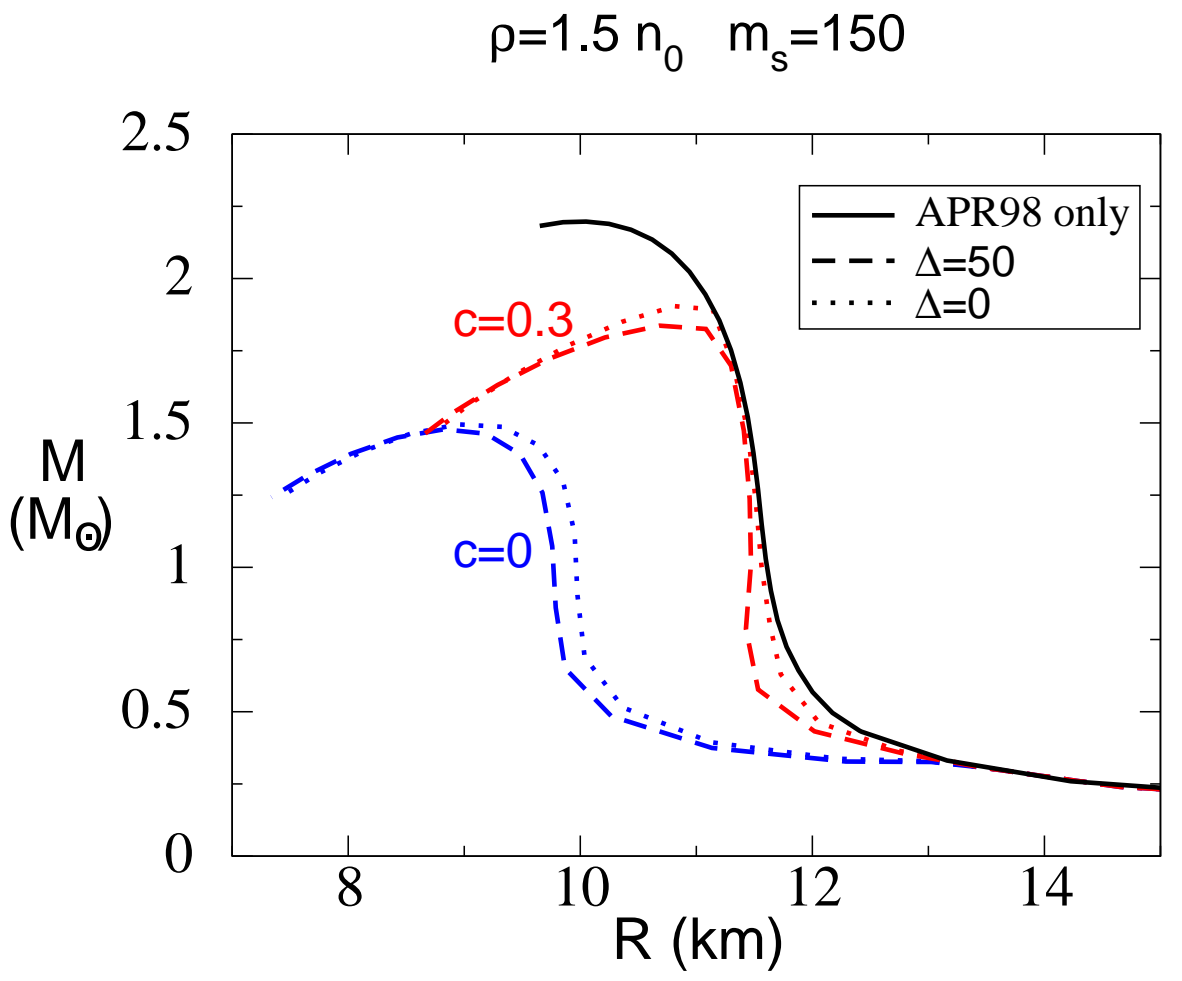

Figure 3: $M(R)$ relationship for APR98 nuclear matter with various quark matter equations of state. The strange quark is light, and the bag constant is tuned so that the nuclear matter to quark matter transition occurs at 1.5 times nuclear saturation density. Dotted lines are unpaired quark matter, dashed lines are CFL with gap of $50 \mathrm{MeV}$. Note how the curve for CFL quark matter with perturbative correction but no colour superconductivity $(c=0.3, \Delta=0$; red dotted) closely follows the pure nuclear curve up to $M \approx 1.8 M_{\odot}$.

\section{$5 \quad$ Other phenomenology}

Mass and size are the most obvious observable properties of a compact star, but various groups are working on other possible signatures of colour-superconducting quark matter. 
Interfaces and mixed phases. These were studied in Ref. [23], and it was found that a mixed phase only occurs if the surface tension of the interface is less than about $40 \mathrm{MeV} / \mathrm{fm}^{2}=0.2 \times(200 \mathrm{MeV})^{3}$, a fairly small value compared to the relevant scales $\Lambda_{\mathrm{QCD}} \approx 200 \mathrm{MeV}, \mu \sim 400 \mathrm{MeV}$. A sharp nuclear-quark interface will have an energy-density discontinuity across it, which will affect gravitational waves emitted in mergers, and also the $r$-mode spectrum and the damping forces to which $r$-modes are subject.

Crystalline pairing (the "LOFF" phase). This is expected to occur when two different types of quark have sufficiently different Fermi momenta that BCS pairing cannot occur [3. This is a candidate for the intermediate phase of Fig. 11, where the strange quark mass, combined with requirements of weak equilibrium and charge neutrality, gives each quark flavour a different Fermi momentum. The phenomenology of the crystalline phase has not yet been worked out, but recent calculations using Landau-Ginzburg effective theory indicate that the favored phase may be a face-centered cubic crystal [24], with a reasonably large binding energy. This raises the interesting possibility of glitches in quark matter stars.

Cooling by neutrino emission. The cooling rate is determined by the heat capacity and emissivity, both of which are sensitive to the spectrum of low-energy excitations, and hence to colour superconductivity . CFL quark matter, where all modes are gapped, has a much smaller neutrino emissivity and heat capacity than nuclear matter, and hence the cooling of a compact star is likely to be dominated by the nuclear mantle rather than the CFL core [25, 26, 27]. Other phases such as 2SC or LOFF give large gaps to only some of the quarks. Their cooling would proceed quickly, then slow down suddenly when the temperature fell below the smallest of the small weak-channel gaps. This behavior should be observable.

$r$-mode spindown. The $r$-mode is a bulk flow in a rotating star that, if the viscosity is low enough, radiates away energy and angular momentum in the form of gravitational waves. One can rule out certain models for compact stars on the grounds that they have such low damping that they could not support the high rotation rates observed in pulsars: $r$-mode spindown would have slowed them down. Madsen [28] has shown that for a compact star made entirely of quark matter in the CFL phase, even a gap as small as $\Delta=1 \mathrm{MeV}$ is ruled out by observations of millisecond pulsars. It remains to extend this calculation to the more generic picture of a quark matter core surrounded by a nuclear mantle.

\section{Acknowledgments}

I thank the organizers of SQM 2003. The work reported in Section 4 was performed in collaboration with Sanjay Reddy, and was supported by the UK PPARC and by the U.S. Department of Energy under grant number DE-FG02-91ER40628. 


\section{References}

[1] M. G. Alford, Ann. Rev. Nucl. Part. Sci. 51 (2001) 131 hep-ph/0102047. K. Rajagopal and F. Wilczek, hep-ph/0011333. T. Schaefer, hep-ph/0304281. D. H. Rischke, nucl-th/0305030. D. K. Hong, Acta Phys. Polon. B 32 (2001) 1253 hep-ph/0101025.

[2] M. Alford, K. Rajagopal and F. Wilczek, Nucl. Phys. B537, 443 (1999) hep-ph/9804403.

[3] M. Alford, J. Bowers and K. Rajagopal, Phys. Rev. D 63, 074016 (2001) hep-ph/0008208.

[4] T. Schäfer, Phys. Rev. D62, 094007 (2000). M. Buballa, J. Hosek and M. Oertel, hep-ph/0204275. M. Alford, J. Bowers, J. Cheyne and G. Cowan, Phys. Rev. D 67, 054018 (2003) hep-ph/0210106.

[5] B. Barrois, Nucl. Phys. B129 (1977) 390; "Nonperturbative effects in dense quark matter", Cal Tech PhD thesis, UMI 79-04847-mc (1979). S. Frautschi, Proceedings of workshop on hadronic matter at extreme density, Erice 1978. D. Bailin and A. Love, Phys. Rept. 107 (1984) 325, and references therein. M. Iwasaki, T. Iwado, Phys. Lett. B350, 163 (1995); M. Iwasaki, Prog. Theor. Phys. Suppl. 120, 187 (1995)

[6] M. Alford, K. Rajagopal and F. Wilczek, Phys. Lett. B422, 247 (1998) hep-ph/9711395. R. Rapp, T. Schäfer, E. V. Shuryak and M. Velkovsky, Phys. Rev. Lett. 81, 53 (1998) hep-ph/9711396.

[7] J. Bardeen, L. Cooper, J. Schrieffer, Phys. Rev. 106, 162 (1957); Phys. Rev. 108, 1175 (1957)

[8] T. Schäfer, F. Wilczek, Phys. Rev. Lett. 82, 3956 (1999) hep-ph/9903503.

[9] N. Evans, J. Hormuzdiar, S. Hsu, M. Schwetz: Nucl. Phys. B581, 391 (2000) hep-ph/9910313.

[10] T. Schäfer, Nucl. Phys. B575, 269 (2000) hep-ph/9909574. I. Shovkovy, L. Wijewardhana, Phys. Lett. B470, 189 (1999).

[11] R. D. Pisarski and D. H. Rischke, "Why color-flavor locking is just like chiral symmetry breaking". To be published in, Proceedings of the Judah Eisenberg Memorial Symposium, "Nuclear Matter, Hot and Cold", Tel Aviv, April 14 16, 1999 nucl-th/9907094.

[12] M. Alford, J. Berges and K. Rajagopal, Nucl. Phys. B558, 219 (1999) hep-ph/9903502. 
[13] M. Alford and K. Rajagopal, JHEP 0206 (2002) 031 hep-ph/0204001.

[14] P. F. Bedaque and T. Schäfer, Nucl. Phys. A 697 (2002) 802 hep-ph/0105150.

[15] K. Iida and G. Baym, Phys. Rev. D 63, 074018 (2001) hep-ph/0011229.

[16] K. Rajagopal and F. Wilczek, Phys. Rev. Lett. 86, 3492 (2001) hep-ph/0012039.

[17] A. W. Steiner, S. Reddy and M. Prakash, Phys. Rev. D 66, 094007 (2002) hep-ph/0205201.

[18] S. L. Shapiro and S. A. Teukolsky, "Black Holes, White Dwarfs, And Neutron Stars: The Physics Of Compact Objects," New York, USA: Wiley (1983) 645 p. J. M. Lattimer and M. Prakash, Phys. Rept. 333 (2000) 121 astro-ph/0002203. H. Heiselberg and V. Pandharipande, Ann. Rev. Nucl. Part. Sci. 50 (2000) 481 astro-ph/0003276.

[19] N. K. Glendenning, "Compact Stars: Nuclear Physics, Particle Physics, And General Relativity," New York, USA: Springer (1997) 390 p.

[20] M. Alford and S. Reddy, Phys. Rev. D 67, 074024 (2003) nucl-th/0211046.

[21] J. Cottam, F. Paerels, M. Mendez, Nature 420, 51 (2002).

[22] E. S. Fraga, R. D. Pisarski and J. Schaffner-Bielich, Phys. Rev. D63, 121702 (2001) hep-ph/0101143.

[23] M. G. Alford, K. Rajagopal, S. Reddy and F. Wilczek, hep-ph/0105009.

[24] J. A. Bowers and K. Rajagopal, hep-ph/0204079

[25] D. Page, M. Prakash, J. Lattimer, A. Steiner, Phys. Rev. Lett. 85 (2000) 2048 hep-ph/0005094.

[26] I. Shovkovy, P. Ellis astro-ph/0207346.

[27] P. Jaikumar, M. Prakash and T. Schafer, Phys. Rev. D 66, 063003 (2002) astro-ph/0203088.

[28] J. Madsen Phys. Rev. Lett. 85, 10 (2000) astro-ph/9912418. 